\title{
BERGSON ET LES DROITS DE L'HOMME: LA CONVERSION ET LE SOUCI DE SOI
}

\author{
Alexandre Lefebure \\ University of Sydney
}

\begin{abstract}
This article examines Henri Bergson's conception of human rights [les droits de l'homme] in The Two Sources of Morality and Religion (1932). I claim that he provides an original view of human rights. Rather than understand human rights primarily as an institution to protect all human beings from serious social, legal, and political abuse, Bergson conceives of them as a medium of personal transformation. In particular, I argue that for him the true potential of human rights is to initiate all human beings into a way of life open to love and joy.
\end{abstract}

Keywords: Bergson; Two Sources of Morality and Religion; Human rights; Care of the self

Resumo: Este artigo examina a concepção bergsoniana de direitos humanos [les doits de l'homme] em As Duas Fontes da Moral e da Religião (1932). Proponho que Bergson apresenta uma visão original quanto aos direitos humanos. Mais do que entendê-los como um dispositivo para proteger os seres humanos de sérios abusos sociais, legais e políticos, Bergson os concebia como um meio de transformação pessoal. Em particular, argumento que para Bergson o verdadeiro potencial dos direitos humanos é iniciar toda a humanidade numa vida predisposta ao amor e à alegria.

Palavras-chave: Bergson, Duas Fontes da Moral e da Religião, Direitos Humanos, Cuidado de si.

Les paradoxes et contradictions de la morale dynamique s'évanouissent si l'on considère l'intention de ses maximes, qui est d'induire un état d'âme.'

Vladimir Jankélévitch, Henri Bergson

Il est difficile de situer Henri Bergson dans la tradition des droits de l'homme. En matière de contribution concrète aux institutions internationales des droits de l'homme, il est unique parmi les grands philosophes. Après la première guerre mondiale, il a travaillé étroitement avec l'administration de Woodrow Wilson pour instaurer la Société des Nations, et il deviendra plus tard président de sa Commission internationale de coopération intellectuelle 
(CICI, la future UNESCO en 1946) ${ }^{1}$. Bien qu'ils ne se soient jamais rencontrés, Bergson a eu une profonde influence sur John Humphrey, qui fut le rédacteur principal de la version préliminaire de la Déclaration universelle des droits de l'homme².

Pourtant, en termes de contribution à la théorie et la philosophie des droits de l'homme, l'impact de Bergson semble négligeable. D’une part, il est presque inconnu par les spécialistes contemporains des droits de l'homme (notamment dans la tradition anglo-américaine). D'autre part, et jusqu'à tout récemment, il n'y avait que très peu de commentaires consacrés à ses observations sur les droits de l'homme ${ }^{3}$. À mon avis, cela est fort regrettable. Dans cet article, j'avancerai que Bergson nous offre une façon tout à fait nouvelle de réfléchir sur les droits de l'homme.

De nos jours, il est généralement admis que les droits de l'homme sont une institution mise en place pour protéger tous les humains des abus les plus graves. Cette hypothèse est tellement intériorisée qu'elle sous-tend pratiquement toutes les grandes théories contemporaines ${ }^{4}$. Bergson, cependant, part d'un point de vue différent. En plus d'accorder aux droits de l'homme le rôle de protéger les humains, Bergson en fait un moyen de transformation personnelle. Ou, pour être plus précise, ma thèse est que pour Bergson le but principal des droits de l'homme est d'initier tous les humains à l'amour.

Cet essai a deux objectifs. D’abord, reconstituer la théorie des droits de l'homme de Bergson. Ensuite, suggérer qu'elle ouvre de nouvelles voies pour la recherche sur les droits de l'homme. Il s'agit là d'un point important. À mon avis, la principale découverte de la théorie de Bergson est de nous amener à reconsidérer à la fois le but et l'objet des droits de l'homme. Comme je l'ai

\footnotetext{
1 Voir Soulez, Bergson politique, 89-126, Soulez et Worms, Bergson, 153-170, Lefebvre et White, 'Introduction.'

2 Curle, Humanité: 'Humphrey tenait un journal intime au début de son mandat aux Nations unies. À travers ses écrits, on peut clairement voir qu'il en est venu à envisager la Déclaration universelle sous l'angle du livre de Bergson Les deux sources' (6). Cependant, pour préciser, ceci est une appréciation rétrospective par Humphrey. II commençait tout juste à lire Bergson en décembre 1948, le même mois où la Déclaration universelle des droits de l'homme fut adoptée par l'Assemblée générale

${ }^{3}$ Lefebvre, Human Rights as a Way of Life: on Bergson's Political Philosophy.

${ }_{4}^{4}$ Prenons, par exemple, les trois principales perspectives anglo-américaines sur les droits de l'homme aujourd'hui: le libéralisme, l'approche des 'capabilités' et le droit international. Même si elles ne s'accordent pas sur ce que les droits de l'homme sont censés protéger - essentiellement: la liberté personnelle pour les libéraux, les capacités 'centrales' pour les théoriciens des capabilités et la dignité humaine pour les tenants du droit international - elles s'accordent toutes sur la mission fondamentale: protéger. Pour la position libérale, consulter Ignatieff, Human Rights as Politics and Idolatry, pour l'approche des capabilités, voir Nussbaum, Creating Capabilities, et pour les instruments juridiques, voir Kateb, Human Dignity and Rosen, Dignity.
} 
déjà laissé entendre, Bergson dévoile un but tout à fait essentiel, mais largement négligé, des droits de l'homme : convertir les êtres humains en plus de les protéger. Par ailleurs, comme Bergson considère les droits de l'homme en termes d'amour et d'introduction à l'amour, ils sont pour lui un instrument de transformation de soi. Autrement dit, pour Bergson, le souci de soi est un objectif des droits de l'homme tout autant que la préoccupation pour autrui. Bien évidemment, il ne s'agit pas de dire que Bergson remet en question le fait que les droits de l'homme essaient de protéger et de prendre soin de tous les êtres humains. Cependant, selon lui, ils ont également d'autres aspects. C'est en nous faisant prendre conscience de ces autres dimensions (celle de la conversion et de celle de la transformation personnelle) que Bergson peut, à juste titre, être considéré comme un théoricien et un praticien exceptionnel des droits de l'homme.

\section{Les droits de l'homme comme religion}

De prime abord, il semble douteux que Bergson présente une analyse des droits de l'homme. Bien sûr, il suggère quelques idées, peut-être même un bref aperçu. Mais une analyse? Les lecteurs familiers avec Les deux sources de la morale et de la religion (1932) trouveront peut-être mon insistance sur les droits de l'homme peu orthodoxe. En effet, même en étant généreux, la discussion explicite des droits de l'homme se limite à environ une douzaine de pages disséminées à travers tout le livre ${ }^{5}$.

Un de mes principaux objectif est toutefois de démontrer que, pour Bergson, les droits de l'homme ne sont pas seulement l'objet d'un intérêt particulier et limité : ils ne représentent pas un sujet parmi tant d'autres et l'importance qu'ils ont pour lui ne correspond pas du tout au peu d'attention directe qu'il leur consacre. Au lieu de cela, les droits de l'homme se trouvent au cœur de sa pensée politique. Je veux dire par là deux choses. D’une part, parce que c'est l'institution politique qui représente le mieux son idéal 'd'amour' et de 'société ouverte' nous verrons que Bergson fonde un immense espoir sur les droits de l'homme. D'autre part, parce qu'ils incarnent cet idéal, nous verrons que Bergson se sert des droits de l'homme comme mesure d'évaluation de toutes les autres institutions et structures politiques. En fait, j'estime que les droits de l'homme dans les Deux sources ont exactement le même statut que la république dans la République de Platon ou que la démocratie dans le Traité théologico-politique de Spinoza : certes, les droits de l'homme sont une institution spécifique, mais, au-delà de ça, ils sont aussi le moyen qui permet d'apprécier le sens, la valeur et l'orientation de tous les autres systèmes politiques.

${ }^{5}$ Les Deux sources de la morale et de la religion, 1000-1100, 1035-1039, 1040-1042, 1215-1216. 
Pour développer cette affirmation, je commencerai par une déclaration audacieuse. Dans les termes de Bergson, les droits de l'homme sont religieux. Ils ne sont pas - comme pour Carl Schmitt ${ }^{6}$ - un concept théologique sécularisé. Il faudrait plutôt dire qu’ils sont religieux et bénéficient du même rapport au divin que, par exemple, l'Église catholique. Cette affirmation surprenante repose sur la définition de la religion de Bergson:

\begin{abstract}
Nous nous représentons donc la religion comme la cristallisation, opérée par un refroidissement savant, de ce que le mysticisme vint déposer, brûlant, dans l'âme de l'humanité. Par elle, tous peuvent obtenir un peu de ce que possédèrent pleinement quelques privilégiés. ... Il y a une vulgarisation noble, qui respecte les contours de la vérité scientifique, et qui permet à des esprits simplement cultivés de se la représenter en gros jusqu'au jour où un effort supérieur leur en découvrira la signification. Du même genre nous parait être la propagation de la mysticité par la religion. En ce sens, la religion est au mysticisme ce que la vulgarisation est à la science (DS 1177-1178).
\end{abstract}

Bergson énonce ici ce qu'il croit être l'essence de la religion, sortie du cadre de toutes ses manifestations historiques. Elle présente trois caractéristiques. D'abord la religion puise ses racines dans une sorte d'amour qui est sans préférence, sans exclusion, et même sans objet. Il appelle cet amour ouvert le 'mysticisme'. Deuxièmement, le rôle de la religion est de 'cristalliser' le mysticisme. Il transforme le mysticisme en une série plus ou moins systématique de règles, de doctrines, de maximes et d'histoires. Troisièmement, l'objectif de la religion est de présenter à 'tous' un mysticisme qu'ils n'auraient peut-être pas rencontré autrement. Elle propage le mysticisme. Ensemble, ces trois caractéristiques définissent ce qui est véritablement religieux dans la religion.

Je me sers de cette définition comme point de départ. Pourquoi? Parce que les droits de l'homme y correspondent point par point. Ils proviennent de l'amour; ils se cristallisent dans une suite de principes, de règles et de symboles; et ils servent à initier tous les humains à l'amour ouvert. Par conséquent, en ce sens, il convient de les appeler religieux.

\title{
Actes d'amour
}

Quelle récompense peut-on attendre de cette assertion potentiellement controversée ? Mon but n'est certainement pas de m'aventurer dans un débat sur la relative 'religiosité' des droits de l'homme ${ }^{7}$. Mon objectif

\footnotetext{
${ }^{6}$ Schmitt, Théologie Politique, chapitre 2.

7 Pour un choix parmi l'abondante littérature à ce sujet, voir Witte et Green (eds.), Religion and Human Rights: an Introduction; Banchoff et Wuthnow (eds.), Religion and the Global Politics of Human Rights; Ignatieff, Human Rights as Politics and Idolatry, 53-95, avec aussi la réponse que lui fait Orentlicher's
} 
est plutôt opportuniste. Comme j’ai déjà dit, même si Bergson nous a laissé une série d'alléchantes remarques sur les droits de l'homme, elles sont franchement trop peu nombreuses. Cependant si je peux établir de façon plausible que les droits de l'homme sont une espèce de mysticisme, alors il me devient possible d'utiliser son analyse détaillée de la religion pour y puiser un concept substantiel des droits de l'homme.

Considérons la première caractéristique distinctive de la religion: ses racines qui plongent dans le mysticisme et l'amour ouvert. Bergson est parfaitement clair: ce genre d'amour est le moteur non seulement des religions historiques mais aussi d'institutions qu'on appelle lä̈ques comme les droits de l'homme et la démocratie. Par exemple, en ce qui concerne la démocratie, il affirme, 'la fraternité est l'essentiel: ce qui permettrait de dire que la démocratie est d'essence évangélique, et qu'elle a pour moteur l'amour' (DS 1215). Selon Bergson, donc, l'amour n'est pas une composante optionnelle et facultative de la politique et encore moins une détérioration potentielle de sa (prétendue) nature rationnelle. Il n'est pas non plus un sentiment qui pourrait être limité à ce qu'on aimerait appeler (et confiner à) une expérience personnelle ou psychologique. Au lieu de cela, c'est une véritable force 'capable de [se] cristalliser en représentations, et même en doctrine' (DS 1015).

L'idée que l'amour puisse être inventif dans un sens emphatique qu'il puisse être 'à l'origine des grandes créations', c'est-à-dire qu'il puisse non seulement inspirer des systèmes politiques, moraux et métaphysiques mais aussi les façonner (DS 1011) - est cruciale. À mes yeux, c'est le point fondamental, le cœur de la théorie de Bergson sur la religion et aussi sur les droits de l'homme. Présentons-le comme ceci: la plupart du temps, Bergson dit que les émotions ne sont pas créatrices. Elles sont plutôt la conséquence d'une représentation, une agitation qui en découle (DS 1011, 1190). Un souvenir douloureux, par exemple, inspire le regret; l'anticipation d'une agréable soirée, le plaisir. L'idée est que, généralement, la représentation précède et génère l'émotion. Comparons maintenant avec ce que Bergson appelle l'émotion créatrice' (DS 1028, 1056, 1192). Dans ce cas, l'émotion ne provient pas d'une représentation. En fait, c'est le contraire: '[L'émotion créatrice] est grosse de représentations, dont aucune n'est proprement formée, mais qu'elle tire ou pourrait tirer de sa substance par un développement organique' (DS 1012). C'est cette deuxième sorte d'émotion qui peut 'se cristalliser' dans une doctrine ou une œuvre d'art. Vers la fin des Deux sources Bergson offre l'exemple suivant:

'Relativism and Religion'; Moyn, The Last Utopia, 11-20; et Glendon, The Forum and the Tower, 199220. 
Quoi de plus construit, quoi de plus savant qu'une symphonie de Beethoven? Mais tout le long de son travail d'arrangement, de réarrangement et de choix, qui se poursuivait sur le plan intellectuel, le musicien remontait vers un point situé hors du plan pour y chercher l'acceptation ou le refus, la direction, l'inspiration : en ce point siégeait une indivisible émotion que l'intelligence aidait sans doute à s'expliciter en musique, mais qui était elle-même plus que musique et plus qu'intelligence. ... Pour en référer à elle, l'artiste avait chaque fois à donner un effort, comme l'œil pour faire reparaître une étoile qui rentre aussitôt dans la nuit. (DS 1190)

Pour développer l'idée d'émotion créatrice, illustrons-la de façon plus directe. Supposons que Beethoven soit en train d'écrire le mouvement final de la Symphonie n.9, 'Ode à la joie'. Supposons de plus, que l'émotion derrière elle est effectivement la joie. Mais déjà nous devons nous arrêter. Bergson s'empresse de souligner que le mot 'joie' est seulement une référence pratique. Ce n'est pas comme si Beethoven se donne pour objectif d'évoquer une émotion déjà connue et éprouvée. 'Ce serait,' dit Bergson 'oublier que joie, tristesse, pitié, sympathie sont des mots exprimant des généralités auxquelles il faut bien se reporter pour traduire ce que la musique fait éprouver, mais qu'à chaque musique nouvelle adhèrent des sentiments nouveaux, créés par cette musique et dans cette musique, définis et délimités par le dessin même, unique en son genre, de la mélodie ou de la symphonie' (DS 1009). Quand Bergson suggère que derrière chaque grande doctrine ou œuvre d'art existe une émotion créatrice, le mot créatif doit donc se comprendre de deux manières différentes. Oui, il indique que l'émotion crée l'œuvre dont on parle. Mais il suggère aussi que l'émotion en elle-même est novatrice. Les émotions, dans ce sens, sont ouvertes. Elles peuvent être classées comme appartenant à une espèce - par exemple 'joie,' 'tristesse,' 'pitié,' ou 'amour' - à la condition qu'on reconnaisse que chacune des grandes émotions apportera une autre dimension à sa catégorie et transformera celle-ci dans son ensemble.

Pour revenir au point principal: la force motrice qui a engendré la création de la Symphonie n.9 est la joie. Mais là aussi nous devons faire attention. Comme Bergson le précise, ce n'est pas un aller simple de l'émotion à la représentation. Ce n'est pas comme s'il y avait une émotion bien définie que Beethoven n'aurait eu qu'à traduire en musique dans un souffle d'inspiration. Ce qui se produit plutôt, c'est un va-et-vient constant entre l'inspiration et sa représentation. Un effort constamment répété est nécessaire. Ici, cependant, le langage de Bergson est potentiellement trompeur. Il semblerait que, comme une 'étoile' que notre œil doit faire 'réapparaitre' dans un ciel de nuit, l'émotion est ressentie tout d'un coup avant sa représentation. En d'autres termes, c'est comme si l'émotion existait hors de son actualisation dans l'œuvre et nécessitait seulement d'être consultée pour de nouveaux 
conseils. Ceci n'est pas le cas. Tout au long des Deux sources, Bergson dit clairement que tout en engendrant la représentation, l'émotion créatrice est simultanément créée par ces représentations. C'est un processus de détermination mutuelle. C'est dans le va-et-vient entre l'émotion et la représentation - ou, dans le langage de Bergson, les allées et venues entre une émotion indivisible et 'le plan intellectuel' - que l'émotion et sa représentation se construisent et se définissent l'une par rapport à l'autre. Donc, quoique les 'sentiments nouveaux' créent indubitablement la musique, il est aussi incontestable que le sentiment existe lui-même 'créés par cette musique et dans cette musique' (DS 1009). Le paradoxe de l'émotion créatrice est que l'œuvre crée l'émotion en même temps qu'elle l'évoque.

Je m'attarde sur ce processus de création artistique afin de présenter comment Bergson conçoit la genèse de la religion et des droits de l'homme. Dans ce cas, cependant, il ne s'agit pas de l'interaction entre joie et musique; ce dont il est question ici c'est l'amour et la loi. Pour illustrer les ressemblances frappantes entre la description de Bergson de la genèse de la religion et celle de la genèse des droits de l'homme, il est instructif de comparer deux passages clefs. Le premier décrit les origines du christianisme, le deuxième celles des 'droits de l'homme':

La morale de l'Évangile est essentiellement celle de l'âme ouverte: n'a-t-on pas eu raison de faire remarquer qu'elle frise le paradoxe, et même la contradiction, dans les plus précises de ses recommandations? Si la richesse est un mal, ne nuirons-nous pas aux pauvres en leur abandonnant ce que nous possédons? ... Mais le paradoxe tombe, la contradiction s'évanouit, si l'on considère l'intention de ces maximes, qui est d'induire un état d'âme. Ce n'est pas pour les pauvres, c'est pour lui que le riche doit faire abandon de sa richesse... Il faudrait alors, en toute rigueur, une expression directe du mouvement et de la tendance; mais si l'on veut encore - et il le faut bien - les traduire dans la langue du statique et de l'immobile, on aura des formules qui frôleront la contradiction. (DS 1024-25) [La justice des droits de l'homme] n'évoque plus des idées de relation ou de mesure, mais au contraire d'incommensurabilité et d'absolu. Cette justice ne comporterait une représentation complète 'qu'à l'infini' comme disent les mathématiciens; elle ne se formule précisément et catégoriquement à un moment déterminé, que par des interdictions; mais, dans ce qu'elle a de positif, elle procède par des créations successives, dont chacune est une réalisation plus complète de la personnalité, et par conséquent de l'humanité. (DS 1037)

Selon Bergson, qu'ont en commun le christianisme et les droits de l'homme? Commençons par le plus évident: les deux cherchent à 'ouvrir ce qui était clos 'et à ' ramener [le groupe] chaque fois à l'humanité' (DS 1203). C'est leur mission essentielle. D'accord, le christianisme et les droits de l'homme ne représentent pas les seuls efforts accomplis au cours de l'histoire pour essayer d'aller au-delà du groupe. Et ils ne seront pas les derniers non plus (DS1039). 
Néanmoins, ils représentent pour Bergson les deux incarnations les plus complètes de l'aspiration vers l'ouverture.

Peut-être encore plus important que cette ambition mutuelle, le christianisme et les droits de l'homme s'accordent aussi sur la manière de la réaliser. Les deux espèrent que la tendance vers l'ouverture s'accomplira en faisant naître, en tous et en chacun, ce que Bergson appelle 'un état d'âme'. Bien entendu, il m'apparait particulièrement important de défendre cet argument dans le contexte des droits de l'homme. Dans le passage ci-dessus, Bergson affirme sans équivoque que le but des enseignements, règles et paraboles de l'Évangile est 'd'induire un état d'âme'. En effet, ce n'est qu'en gardant l'œil sur ce but que ces enseignements prendront leur sens et leur éviteront de tomber dans le paradoxe et la contradiction. ${ }^{8}$

'La morale de l'Évangile' et 'les droits de l'homme' partagent donc plusieurs choses: l'origine (amour ouvert ou mysticisme), le but (ouvrir ce qui était fermé) et la méthode (induire un état d'âme). Leur lien le plus profond, toutefois, concerne le statut et le rôle des représentations qu'ils engendrent: maximes, règles et histoires pour le christianisme; normes, lois et mythes pour les droits de l'homme. Tout cela constitue un champ de la pensée de Bergson particulièrement difficile et dont l'origine remonte très loin. En effet, le problème de la représentation de l'amour renouvelle ce qui est, peut-être, la plus ancienne problématique de l'œuvre de Bergson: la relation entre le spirituel' et la réalité temporelle (incluant le mouvement, la vie intérieure et l'évolution) d'une part et d'autre part nos tentatives pour le représenter.

Pour s'en convaincre vous n'avez qu'à regarder le titre de son premier livre! - Essai sur les données immédiates de la conscience. Ici, comme le dit Suzanne Guerlac, 'Bergson nous demande de considérer un niveau d'expérience qui est immédiate et qui ne passe pas par le langage ou par une notation quantitative une expérience du 'vrai qui résiste à la symbolisation." Ainsi donc commence le paradoxe au cœur de l'œuvre de Bergson: exprimer quelque chose, qui, par sa nature intrinsèque, défie la représentation, comme 'la durée', 'les multiplicités continues', 'le passé pur', 'l'élan vital', 'l'émotion créatrice', la liste est longue. Chacun de ces concepts a été créé afin de désigner ce que la pensée représentationnelle ne peut atteindre ou ce qu'elle tente d'éviter.

Mais 'l'amour ouvert' ou 'le mysticisme' forment peut-être une catégorie à part. Ce concept n'essaie pas seulement de capter une autre sorte d'émotion créatrice qui tout à la fois provoque et inspire la représentation, tels que la joie, l'admiration ou l'amour chevaleresque (DS 1009-10). Les concepts

8 Voir Jankélévitch, Henri Bergson, 191.

${ }_{9}$ Guerlac, Thinking In Time, 43. 
d'amour ouvert et de mysticisme de Bergson s'attachent plutôt, si l'on peut dire, à quelque chose de particulièrement impossible à représenter! Je m'explique: le concept de l'amour ouvert ou du mysticisme combine toutes les réalités extra-représentatives que les autres concepts de Bergson. En tant qu'émotion créatrice, il précède la représentation; en tant que durée, il est une expérience psychique indivisible; en tant que passé pur, il est sans objets distincts; et en tant qu'élan vital, il est le mouvement et la créativité de la vie elle-même ${ }^{10}$. Nous pouvons donc voir la tâche extraordinaire que Bergson assigne à la religion et, je soutiens, aux droits de l'homme: créés par l'amour et cherchant à initier tout le monde à l'amour, la religion et les droits de l'homme doivent proposer ce qui défie la représentation sur plusieurs plans.

Par conséquent, quand Bergson déclare que la justice des droits de l'homme n'est représentée 'que par des interdictions', ou quand il énonce que 'la langue du statique et de l'immobile' est nécessaire pour représenter la moralité de l'Évangile, il ne veut pas dire que ces symbolisations représentent certains aspects de la justice ou couvrent certains éléments de l'Évangile. Ce n'est pas comme si quelques parties de la moralité ouverte pouvaient trouver un mode d'expression dynamique alors que d'autres seraient condamnées à recevoir un traitement négatif ou statique. Son propos, au contraire, est que ces représentations traduisent le tout de la justice 'absolue' (dans le cas des Déclarations des droits de l'homme) et le tout de la moralité ouverte (dans le cas de l'Évangile). Ces représentations sont donc, dans un sens, le contenu entier de cette sorte de justice et de moralité. Toutefois, en un autre sens, il est clair que pour Bergson ces représentations ne sont pas indispensables, elles constituent des préliminaires et sont d'une certaine façon expéditives. Évidemment, l'Évangile et la Déclaration des droits de l'homme actualisent une émotion créatrice. Et bien sûr, ils lui servent d'introduction. Mais pour autant, ils demeurent absolument distincts et différents de l'amour qui les inspire.

Comme représentations d'amour ouvert, la religion comme les droits de l'homme accomplissent quatre choses à la fois: (1) ils expriment l'amour, (2) ils définissent l'amour, (3) ils obscurcissent l'amour et (4) ils nous initient à l'amour. Chacun de ces différents éléments trouve sa place dans la remarque suivante: '[Les mystiques] voudraient entraîner avec eux l'humanité; ne pouvant communiquer à tous leur état d'âme dans ce qu'il a de profond, ils le transposent superficiellement; ils cherchent une traduction du dynamique en statique, que la société soit à même d'accepter et de rendre définitive par l'éducation' ( $D S$ 1208). Cette remarque est utile pour résumer les thèmes

10 Voir Lefebvre, Human Rights as a Way of Life, 90-109. 
principaux de cette section. L'amour ouvert ou mystique est une émotion créatrice. Il engendre sa propre représentation, notamment ces deux exemples que sont 'la moralité de l'Évangile' et les 'droits de l'homme'. Ces deux représentations expriment l'amour ouvert. Plus encore, elles arrivent à définir son contenu. L'amour ouvert, par conséquent, adopte des contours précis dessinés par les normes, les principes et les mythes, conformément à toutes les représentations contenues dans l'Évangile et les droits de l'homme.

Dans un même temps, ces représentations déforment et masquent pourtant la vraie nature de l'amour ouvert. Elles transforment un amour sans objet, et qui incarne le mouvement et la créativité de la vie elle-même, en une série de 'recommandations' (dans le cas de l'Évangile) ou 'd'interdictions' (dans le cas de la Déclaration). En d'autres mots, elles convertissent l'amour ouvert en un système d'obligations et de devoirs dont il diffère absolument. Comme le dit Bergson, 'l'aspiration morale nouvelle ne prend corps qu'en empruntant à la société close sa forme naturelle, qui est l'obligation' (DS 1203). De cette façon, l'amour ouvert donne lieu à une 'religion mixte', composée à la fois de tendances morales ouvertes et fermées (DS 1157). Le christianisme s'apparente à une telle religion. Et les droits de l'homme aussi.

\section{La tendance fermée des droits de l'homme}

Nous venons de souligner que l'amour ouvert ou mystique crée des représentations qui donnent lieu à ce que Bergson appelle une 'religion mixte'. En ce sens, toute religion - et n'oublions pas que ce terme inclut les droits de l'homme - est mixte et déjà mélangée. Aussitôt que l'amour ouvert se transpose en règles, en principes et en mythes, un mélange de tendances morales fermées et ouvertes se crée. Le fait que la religion soit mixte ne dérange pas Bergson. Mais pourquoi? Tout simplement parce que c'est ce que l'amour doit faire pour se transmettre (DS 1158). Ce qui le dérange cependant, c'est notre tendance à oublier, à perdre de vue, et même à nier l'amour ouvert qui est à sa source. Ce dont nous avons besoin est donc de distinguer soigneusement les tendances ouvertes des tendances fermées à l'intérieur des droits de l'homme, et de reconnaitre également qu'elles forment un composé.

Nous avons déjà évoqué la tendance ouverte des droits de l'homme. Sa caractéristique essentielle est un genre d'amour sans exclusion, sans fermeture et sans préférence. Passons maintenant à la tendance fermée. Il pourrait sembler étonnant qu'on puisse retrouver une tendance fermée dans les droits de l'homme. Après tout, n'est-ce pas leur raison d'être que de contrer les dangers des tendances fermées, telles l'exclusion, la xénophobie et la persécution? Oui, bien sûr, et Bergson affirme que les droits de l'homme essaient de retourner cette tendance fermée contre elle-même. En ce sens, ils 
opèrent une dialectique. Les droits de l'homme nient et préservent tout à la fois les aspects importants qui sont au cœur de la tendance fermée.

Mais qu'est-ce que cela signifie? Concrètement, qu’y a-t-il de fermé dans les droits de l'homme? Nous pouvons ici mettre en évidence trois formes caractéristiques du clos.

1. Les droits de l'homme ont exclusivement pour objet les êtres humains.

2. Les droits de l'homme dépendent de regroupements fermés (et en particulier des États) pour leur appui et leur mise en pratique.

3. Les droits de l'homme établissent des mythes fondamentaux (ce que Bergson appelle des 'fabulations') sur les êtres humains.

Commençons par ce qui éloigne, de la façon la plus flagrante, les droits de l'homme de la tendance ouverte de la moralité: le fait de nommer l'objet vers lequel tendra l'intérêt, l'attention particulière et l'amour, en l'occurence les êtres humains. Deux sortes de fermetures en résultent. Bien évidemment, l'interprétation classique des droits de l'homme fait une distinction entre humains et animaux en termes de dignité, de caractère sacré et de protection. Compte-tenu du fait qu'on a déjà largement et abondamment traité de cette division dans la littérature sur les droits de l'homme et ailleurs, je n'en parlerai pas ici ${ }^{11}$. Cependant une deuxième forme d'exclusion mérite notre attention. Les droits de l'homme, et plus précisément les concepts et les institutions qui sont au cœur de son cadre conceptuel, comme le 'génocide', 'les crimes de guerre', et 'les crimes contre l'humanité, contribuent aussi à établir une division au sein même de l'humanité, entre les humains (la grande majorité) et ces autres individus qui, en quelque sorte, ont renié leur humanité et n'appartiennent plus au genre humain.

Considérons les dernières lignes accablantes du livre de Hannah Arendt, Eichmann à Jérusalem. Ce sont, dit-elle, les mots que les juges israéliens d'Adolf Eichmann auraient osé adresser s'ils avaient donné suite aux motifs de leur arrêt.

Vous (Adolf Eichmann) avez raconté votre histoire comme celle de quelqu'un qui n'a pas eu de chance, et, connaissant les circonstances, nous sommes prêts à vous accorder, jusqu'à un certain point du moins, que si vous aviez bénéficié de circonstances plus favorables, vous n'auriez probablement jamais eu à comparaitre devant nous ou devant une autre cour pénale. Supposons, pour les besoins de la cause, que seule la malchance a fait de vous

11 Les ouvrages classiques écrits sur le sujet: Singer, Animal Liberation, and Regan, The Case for Animals Rights. Plus récemment, nous pourrions ajouter Nussbaum and Sunstein (eds.), Animal Rights; Kateb, Human Dignity, et Crary, 'Human, Animals, Right and Wrong.' 
un instrument consentant dans l'organisation du meurtre de masse; il reste encore le fait que vous avez exécuté, et donc soutenu activement, une politique de meurtre de masse. Car la politique et l'école maternelle ne sont pas la même chose ; en politique obéissance et soutien ne font qu'un. Et puisque vous avez soutenu et exécuté une politique qui consistait à refuser de partager la terre avec le peuple juif et les peuples d'un certain nombre d'autres nations comme si vous et vos supérieurs aviez le droit de décider qui doit et ne doit pas habiter le monde - nous estimons qu'on ne peut attendre de personne, c'est-à-dire d'aucun membre de l'espèce humaine, qu'il veuille partager la terre avec vous. C'est pour cette raison, et pour cette raison seule, que vous devez être pendu ${ }^{12}$.

Ce qui rend ce passage renversant n'est pas le verdict qu'il prononce mais la voix avec laquelle il s'exprime. Ici, bien sûr, Eichmann n'est pas jugé par un individu particulier (i.e., Hannah Arendt). Mais il n'est pas non plus condamné par un fonctionnaire ou représentant d'un groupe persécuté (i.e. le peuple juif). La condamnation est en fait prononcée par ni plus ni moins qu'un membre de le genre humain. Pourtant, loin d'être une catégorie universelle ou inclusive, cette perspective de la race humaine est adoptée afin d'expulser un individu de ce groupe. Ce 'nous' est donc utilisé afin de faire de l'humanité un groupe fermé et, dans cette perspective, d'exclure et d'exécuter l'individu qui ne veut pas partager la terre avec nous.

Nous pouvons aborder le verdict de la façon suivante. Quelle sorte d'homme est Adolf Eichmann? Dans les termes de Bergson, il est un cas extrême de non-mystique. 'Certains', observe Bergson, 'sans aucun doute, sont totalement fermés à l'expérience mystique, incapables d'en rien éprouver, d'en rien imaginer' ( $D S$ 1184). Dans ce sens, ne pouvons-nous dire qu'un criminel de guerre et le (parfait ou presque parfait) non-mystique sont analogues? Tous deux désignent un individu chez qui - par constitution ou par coutume - la tendance ouverte est négligeable. Comment donc aller à la rencontre de cette personne? Selon le point de vue d'Arendt, ce n'est pas possible. Si la tendance ouverte ne peut toucher certaines personnes, si elles manquent d'un minimum de volonté pour partager la terre avec les autres, et si leurs actions démontrent que l'idée d'une humanité commune leur est inconcevable, alors il devient nécessaire de créer un groupe universel et de les en exclure. Il devient impératif, en d'autres mots, de mettre en place un groupe, et avec lui une institution, qui se fonde sur la même sorte de morale et de pression juridique qui caractérise la société fermée. Les dispositifs internationaux et les

12 Arendt, Eichmann à Jérusalem, 1286-1287. 
mécanismes relatifs aux droits de l'homme qui poursuivent les cas de violations sont précisément de ce genre.

La seconde caractéristique de tendance du 'clos' dans les droits de l'homme est leur dépendance - non seulement en termes de ressources matérielles et symboliques, mais également en termes de mise en œuvre et d'application - aux groupements fermés tels que l'État. Je serai bref sur ce sujet puisque le sujet est bien traité, non seulement dans la littérature sur les droits de l'homme, mais aussi dans un petit nombre de commentaires sur Bergson et les droits de l'homme ainsi que dans quelques remarques de Bergson lui-même ${ }^{13}$. Un résumé de deux lignes sur la structure des Deux sources peut servir d'introduction. Dans les chapitres 1 ('L'obligation morale') et 2 ('La religion statique') Bergson examine la tendance du clos dans la morale et la religion et dans le chapitre 3 ('La religion dynamique'), il se tourne vers la tendance ouverte. Son problème dans le chapitre 4 ('Remarques finales'), cependant, est premièrement de se demander pourquoi, si le mysticisme est déjà apparu dans le monde, la tendance du clos n'a pas été surmontée et, deuxièmement, à la lumière de cet échec, de proposer des techniques supplémentaires ou des moyens dissuasifs pour la réguler et la contrôler ${ }^{14}$. L'objectif fondamental d'une organisation internationale comme la Ligue des nations, dit Bergson, devrait être de renforcer la réglementation de l'humanité (DS 1222).

Même si les détails sur les questions particulières qu'il soulève coopération économique, contrôle de la population - ne nous préoccupent pas (ou tout au moins pas de la même façon qu'ils le préoccupaient), le principe sous-jacent nous intéresse. Si les guerres doivent diminuer, dit Bergson, il est, dans les circonstances actuelles, nécessaire d'établir une organisation internationale (et non pas supranationale) pour surveiller, encadrer, voire même intervenir dans les politiques nationales. 'C'est une erreur dangereuse,' dit-il, 'que de croire qu'un organisme international obtiendra la paix définitive sans intervenir, d'autorité, dans la législation des divers pays et peut-être même dans leur administration' (DS 1222) ${ }^{15}$. Même si le langage de Bergson peut nous sembler à la fois trop innocent et trop radical dans le défi qu'il lance à la souveraineté de l'État, la logique qu'il applique demeure néanmoins présente dans les doctrines contemporaines inspirées par les droits de l'homme (comme la 'responsabilité de protéger' et l'intervention humanitaire) ainsi que dans les

13 Sur les droits de l'homme, voir Engle Merry, Human Rights and Gender Violence (36-102). Sur Bergson, voir Curle, Humanité, 93-99.

14 Worms, Bergson ou les deux sens de la vie, 318.

${ }^{15}$ Voir les remarques de Bergson dans 'Mes missions' sur le rôle de la Ligue des nations, Mélanges, 1565-1566. Voir aussi Soulez et Worms, Bergson, 163-166. 
mécanismes de leur mise en œuvre (comme les organes chargés des droits de l'homme aux Nations Unies et la Cour pénale internationale). Toutes ces doctrines et mécanismes continuent de dépendre de l'association de groupes fermés et visent à exercer une influence (la plupart du temps de façon consensuelle, mais pas toujours) sur la législation et l'administration d'États souverains.

Le dernier élément du clos qui nous concerne est aussi le plus commenté dans les sections des Deux sources, ce que Bergson appelle la fonction fabulatrice de la religion. En bref, Bergson croit que les mythes que nous retrouvons au cœur de chaque tradition religieuse remplissent une fonction (biologique ou évolutive): contrebalancer la déprimante, débilitante et démoralisante puissance de notre intellect. Tout particulièrement, ils servent à contrecarrer trois idées intrinsèques de l'intellect humain.

1. L'intellect nous dit que nous allons mourir (DS 1086). Cela est une idée décourageante.

2. L'intellect conseille l'égoïsme et nous exhorte à nous occuper de nous-mêmes (DS 1078). Cela est une idée dissolvante.

3. L'intellect calcule les résultats et ainsi démontre que l'incertitude est partout (DS 1094). Cela est une idée démoralisante.

Ces trois points sont connus sous l'acronyme des 3D. Comme nous pouvons l'imaginer, ils ne sont pas tellement propices à former ce que Bergson appelle un attachement à la vie! Ils contribuent à mettre l'espèce en danger en introduisant de l'inquiétude dans la vie, de l'égoïsme dans la société, et de l'incertitude dans l'action (DS 1062, 1077, 1112).

Un thème récurrent dans les Deux sources est que la nature évoluée de l'espèce humaine pourrait dans certaines circonstances représenter une menace à notre propre survie. Dans ce cas, nous pouvons voir que l'intellect humain pose problème pour l'évolution. Comment des êtres accablés par de telles idées pourraient-ils s'épanouir dans le monde ? Bergson répond: le concept de 'fabulation' ou plus précisément encore, la 'fonction de fabuler' de l'intellect. La fabulation est le pouvoir de l'intelligence d'engendrer des 'idées' ou des 'mythes' pour contrebalancer les 3D. Bergson affirme tout spécialement que l'intellect crée trois idées principales (i.e. les mythes ou, pour emprunter une formule à Lévi-Strauss, les 'mythèmes') qui, chacune, contrôle un élément des $3 \mathrm{D}$.

1. La fabulation postule 'une continuation de la vie après la mort' (DS 1086). L'âme et l'après-vie sont des exemples d'idées pour contrecarrer la perspective démoralisante de l'inéluctabilité de la mort.

2. La fabulation nous prévient que 'devant la barrière ouverte, un gardien avait surgi, qui interdisait l'entrée et repoussait le contrevenant... [il] 
défendra, menacera, réprimera' (DS 1078). Le karma et le jugement dernier sont des exemples d'idées pour contrecarrer le danger dissolvant posé par l'égoïsme.

3. La fabulation imagine 'des puissances amies' responsables de nos succès et 'des puissances défavorables' responsables de nos échecs (DS 1094). La foi et la Providence sont des exemples d'idées pour contrecarrer l'incertitude d'agir.

Ces trois fabulations sont ce que Bergson appelle 'des idées religieuses élémentaires' (DS 1092). Retrouvées au cœur de chaque tradition religieuse, elles sont une réaction biologique et évolutive aux préoccupations fondamentales de l'intellect humain, d'où leur universalité. 'La vérité', dit Bergson, 'est que la religion, étant coextensive à notre espèce, doit tenir à notre structure' (DS 1125).

Deux conclusions peuvent être tirées de cette extraordinaire déduction biologique de la religion. D'abord, les convictions religieuses essentielles ne sont pas fondamentalement opposées à la raison ou à l'intelligence. Elles sont plutôt ce qui permet aux êtres humains d'être dans le monde avec leur intellect. Sans le pouvoir compensateur de la fabulation, l'intellect serait insupportable. '[Comme] une réaction défensive de la nature contre ce qu'il pourrait y avoir de déprimant pour l'individu, et de dissolvant pour la société,' les idées religieuses élémentaires laissent littéralement les humains vivre avec leur intelligence ( $D S$ 1150).

Ensuite, même s'il emploie des termes comme 'mythe' et 'fabulation', les idées religieuses élémentaires ne sont ni fausses ni absurdes. Afin de décrire leur statut de vérité, Bergson invente un terme qui semble paradoxal: fabulation véridique (DS 1204). Il ne fait aucun doute qu'envisagées dans une perspective orthodoxe de théories de la vérité, de telles idées sont pour le moins suspectes. Mais peut-être ne le sont-elles pas d'un point de vue pragmatique. $\mathrm{Si}$, en se référant à William James, nous acceptons que la fonction primaire de la vérité est d'assurer la cohérence, la stabilité, et la souplesse de nos interactions, alors les convictions religieuses élémentaires répondent à ce critère $^{16}$. En écartant ces inquiétudes fondamentales de l'intellect, elles sauvent l'espèce d'une existence austère, solitaire et remplie d'anxiété. Avec son concept de fabulation, Bergson inverse donc une typologie classique du Siècle des Lumières. Au lieu de réaffirmer le besoin de la raison de faire obstacle à la superstition, il affirme plutôt que le mythe est nécessaire pour contrecarrer l'intelligence. La fabulation, et non l'intellect, est la faculté décisive.

16 James, Pragmatism, 103. 
Jusqu'à un certain point, cependant. Une idée tenace dans les Deux sources, est, qu'à la longue, les fabulations véridiques seront enfouies sous les traditions et les rituels. Ce qui répondait auparavant à un besoin fondamental (i.e. repousser les 3D) se transforme donc, petit à petit, en une multitude de croyances diverses qui éventuellement versent dans l'absurde. Ayant constaté ce processus d'accumulation et de distorsion extrêmes, Bergson explore deux pistes.

D’un côté, il examine la diversification des idées religieuses élémentaires afin de rechercher une étiologie des aspects de la religion qui sont tout à fait irrationnels, comme la magie et la superstition (DS 1115-49). De l'autre, il essaie de retrouver le sens et la fonction originels des idées religieuses élémentaires. Il le fait, en grande partie, en se focalisant sur des expériences soudaines et souvent dramatiques dans lesquelles ces idées élémentaires sont révélées dans toute leur pureté. Ces expériences donnent lieu à quelques-unes des pages les plus pittoresques des Deux sources. William James et le tremblement de terre de San Francisco (DS 1105-9), la femme qui tombe presque dans la cage d'ascenseur (DS 1076), ou Bergson et son état d'esprit quand il apprend la guerre avec l'Allemagne (DS 1110) et quand son cheval s'emballe (DS 1109) - il étudie toutes ces expériences afin d'accéder à une nouvelle idée religieuse élémentaire qui découlerait directement de la nature.

Peut-être y a-t-il une autre façon de rendre manifeste les idées religieuses élémentaires? Bergson dit qu'il faudrait racler des siècles de tradition pour seulement les apercevoir. Et si ces idées religieuses élémentaires étaient encore créées ici et maintenant ? Si c'était vrai, elles proviendraient directement de la nature. Pour l'instant, Bergson a identifié seulement trois idées religieuses élémentaires: l'immortalité (en réponse à la conscience de la mort), le jugement divin (en réponse à la tentation de l'égoïsme) et la main de la Providence (en réponse à nos doutes quant à l'action). Que manque-t-il pour en rajouter une quatrième?

Les Deux sources nous apportent la réponse: une nouvelle idée religieuse élémentaire nécessite une nouvelle découverte dévastatrice de l'intellect. C'est dans ce sens que je propose que la dignité intrinsèque et inhérente à tous les humains est une idée religieuse élémentaire. C'est précisément un mythe ou une fabulation au sens bergsonien. Et le problème auquel elle répond - c'est-à-dire la nouvelle idée démoralisante qu'elle contrecarre - est la superfluité de l'être humain que les formes contemporaines de pouvoir et de violence révèlent.

Les Deux sources n'est peut-être pas le texte le plus approprié pour soutenir ma suggestion selon laquelle la dignité dans les droits de l'homme fonctionnerait comme une fabulation. Ayant écrit avant la Deuxième Guerre 
mondiale, Bergson n'était pas au courant de toutes les atrocités sui generis. Peutêtre vaudrait-il mieux nous tourner vers Origins of Totalitarianism d'Arendt. Dans sa tentative pour comprendre l'horrible revirement de la situation, Arendt est guidée par une seule vision: le totalitarisme est une nouvelle forme de gouvernement qui diffère pas sa nature de la tyrannie. Il y a plusieurs raisons qui expliquent son caractère unique ${ }^{17}$. La plus pertinente pour nous est celle qui est liée à ce qu'Arendt appelle la 'superfluité de l'homme':

Le totalitarisme ne tend pas vers un gouvernement despotique des hommes, mais vers un système dans lequel les êtres humains sont superflus. Le pouvoir total ne peut être achevé et préservé que dans un monde de réflexes conditionnés et de marionnettes ne présentant pas la moindre trace de spontanéité... Le sens commun proteste désespérément en affirmant que les masses sont soumises et que tout ce gigantesque appareil de terreur est donc superflu; s'ils étaient capables de dire la vérité, les dirigeants totalitaires répliqueraient: 'l'appareil ne vous semble superflu que parce qu'il sert à rendre les hommes superflus ${ }^{118}$.

Comment le problème de la superfluité se compare avec les 3D? Il serait juste de dire que les 3D abordent le problème de la finitude humaine. La connaissance de la mort, le caractère ingouvernable de l'égoïsme et l'incertitude de l'action sont autant de façons de lier et de limiter les êtres humains. En ce sens, les idées religieuses élémentaires que Bergson a explicitées sont des défenses contre la finitude. Le totalitarisme, cependant, soulève un type de problème entièrement différent. Il ne démontre pas que la vie humaine est brève, ni que le savoir humain est limité. Il essaie plutôt de montrer que la vie humaine peut être transformée en un rien du tout: la superfluité exprime une existence sans signification, sans possibilité de réclamer quelque chose au monde ou d'offrir quelque chose en retour. Le totalitarisme n'essaie donc pas de mettre à nu l'existence humaine dépouillée de toute illusion; comme l'explique Arendt, il n'entreprend rien de moins que la destruction de la nature profonde de l'homme ${ }^{19}$. Ce qui pose un problème terrifiant et nouveau. Mais, si tel est le cas, cela voudrait dire qu'on ne peut pas compter sur les idées religieuses élémentaires pour contrebalancer l'idée de la superfluité. Elles n'apportent pas de solution à ce problème. Une nouvelle idée devient impérative.

La dignité, peut-être, répond à cet appel. Conçue comme une réaction de défense contre la possibilité que l'existence humaine soit superflue ou nulle,

\footnotetext{
17 Pippen, 'Hannah Arendt and the Bourgeois Origins of Totalitarian Evil.'

${ }^{18}$ Arendt, Les Origines du totalitarisme, p.808-809.

19 Ibid., p.808.
} 
elle serait une nouvelle idée religieuse élémentaire. Il apparait bien sûr primordial de reconnaittre que le concept de dignité précède celui de superfluité. Mais il me semble tout aussi essentiel d'admettre que ce concept est renouvelé ou recréé dans le contexte de ce problème ${ }^{20}$. Lorsqu'on se rend compte qu'avec le totalitarisme 'tout est possible' et que, dorénavant, d'inimaginables nouvelles vulnérabilités de l'être humain pourraient être révélées, la dignité cesse d'être un simple concept désignant la valeur de chaque être humain par rapport à une qualité intrinsèque (comme la conscience ou la rationalité). Elle devient quelque chose de plus: une assertion fondamentale de la valeur incommensurable de chaque vie humaine. De cette manière, la dignité se comporte moins comme un concept désignant une caractéristique de l'être humain et remplit donc mieux le rôle structurel d'une idée religieuse élémentaire : un mythe, qui n'en est pas moins vrai, pour contrecarrer ce qui est malheureusement une nouvelle réalité (i.e., la superfluité), à la fois déprimante pour l'individu et dissolvante pour la société. C'est en ce sens que je suggère que nous découvrons, au cœur du discours des droits de l'homme, l'élément fondamental de la religion statique selon Bergson: la fonction de fabulation de l'intellect incarnée dans le ' mythe' de la dignité humaine.

Évidemment nous n’avons qu'effleuré la surface du concept bergsonien de 'dignité'. Il est également clair que cette description des caractéristiques du 'clos' dans les droits de l'homme n'est qu'une ébauche. Pour le moment, mon intention est simplement de démontrer que les droits de l'homme sont un phénomène mixte, et d'indiquer les façons particulières par lesquelles ils essaient de contourner les tendances clos.

\section{Conclusion}

Pour conclure, j'aimerais revenir sur mes pas et demander quelle est la contribution de Bergson à notre réflexion sur les droits de l'homme. Ma thèse est qu'il nous apporte un changement radical de perspective. Ordinairement, nous considérons les droits de l'homme comme une protection de notre statut d'être humain. Ce qu'ils protègent varie d'une théorie à l'autre. Cela pourrait être la dignité, la liberté, un ensemble de capacités basiques ou quelque chose d'autre - mais on reconnait toujours que le travail des droits de l'homme consiste à protéger les humains. Comme j'ai déjà dit, je ne suggère pas que Bergson conteste ou remette en question ce but traditionnel. Cependant, selon

20 Je pense notamment à la prééminence accordée au concept de dignité dans le premier article de la Déclaration des droits de l'homme (1948) ('Tous les êtres humains naissent libres et égaux en dignité et en droits') et de même que dans la Grundgesetz de la République fédérale d'Allemagne (1949) ('La dignité de l'être humain est intangible. Tous les pouvoirs publics ont l'obligation de la respecter et de la protéger.'). 
sa conception, l'objectif de protection ne se réalisera qu'après la conversion. En ce sens, il fait classe à part.

Mais au-delà de la théorie particulière de Bergson sur les droits de l'homme, je suis frappé par le fait qu'il ouvre une nouvelle problématique: comment les droits de l'homme servent-ils de moyen de transformation personnelle à la lumière de défis politiques aussi considérables qu'urgents?

Nous pourrions identifier provisoirement trois secteurs d'intérêt associés à cette problématique. D’abord, la possibilité de nouvelles lectures des grands penseurs de l'histoire des droits de l'homme. Avec Bergson, j’ai essayé de rendre cette problématique explicite. Mais les coordonnées fondamentales de cette problématique peuvent aussi être retrouvées chez d'autres penseurs, classiques et contemporains, des droits de l'homme, chacun d'eux l'entraînant dans une direction différente. Pour Mary Wollstonecraft, par exemple, les droits de l'homme aident les femmes à se détacher de vertus (comme la beauté et la chasteté) qui sont les instruments mêmes de leur sujétion ${ }^{21}$. Pour Alexis de Tocqueville, c'est à travers l'exercice de leurs droits à la liberté politique que les individus pourront aspirer à modérer des tendances démocratique comme la solitude liée à l'individualisme et l'insatisfaction à l'égard du matérialisme. Pour John Rawls, les concepts comme le voile d'ignorance peuvent être pris comme des exercices spirituels destinés à aider chacun d'entre nous à entrer en adéquation avec le pluralisme des sociétés modernes. La liste, bien sûr, est incomplète et schématique. Ce que je veux dire, c'est qu'à travers Bergson nous prenons davantage conscience d'une préoccupation récurrente, mais qui demeure implicite, pour la transformation personnelle dans la tradition des droits de l'homme.

Les deuxième et troisième domaines d'intérêt proviennent de la rencontre mutuellement enrichissante entre les droits de l'homme et la théorie et la pratique de la transformation de soi. Qu'est-ce que notre interrogation sur les droits de l'homme aurait à gagner de la pratique de la transformation de soi? La récompense immédiate serait de reconnaître que la pratique de la transformation de soi fait partie intégrante. Mais il y a aussi deux autres avantages. À travers les études des philosophies du développement de soi, nous gagnons une meilleure compréhension de l'orientation subjective ou spirituelle présupposée par le discours des droits de l'homme. Par exemple, il semble que la tradition des droits de l'homme partage plusieurs des principaux objectifs de transformation de soi de la période antique, c'est-à-dire le développement de la tolérance, l'impartialité et une perspective universelle. Si

${ }^{21}$ Voir Lefebvre, 'Mary Wollstonecraft, Human Rights, and the Care of the Self' et 'The Rights of Man and the Care of the Self'. 
cela est vrai, il devient possible d'aborder les objectifs des droits de l'homme à travers les traditions antiques qui ont sérieusement et concrètement réfléchi sur ces questions. Il devient aussi possible d'imaginer une autre généalogie des droits de l'homme qui s'inspirerait des discours d'exercices spirituels et de l'ascétisme.

Et maintenant, la question inverse : 'que gagnerait notre réflexion sur la transformation de soi si elle se portait sur les droits de l'homme?' Les bénéfices de cette problématique seraient également substantiels. Surtout, elle permettrait de voir comment la pratique de la transformation de soi se réinvente à travers des problèmes contemporains. Étant donné la primordialité de la transformation de soi dans la philosophie ancienne, il n'est pas surprenant que les grands auteurs contemporains qui écrivent sur ce sujet se penchent principalement sur la période antique. Pourtant, ces mêmes auteurs contemporains déploient tous leurs efforts pour démontrer à quel point le thème de la transformation de soi serait poursuivi par les auteurs modernes. Dans ce contexte, la préoccupation à l'égard des droits de l'homme est exemplaire puisqu'elle montre bien comment la pratique de la transformation personnelle est renouvelée dans la situation actuelle et à travers nos problèmes politiques $^{22}$.

\section{Bibliographie}

ARENDT, Hannah. Les Origines du totalitarisme et Eichmann à Jérusalem, Paris: Gallimard, 2002.

BANCHOFF, Thomas; WUTHNOW, Robert (eds). Religion and the Global Politics of Human Rights. Oxford: Oxford University Press, 2011.

BERGSON, Henri. Mélanges. Paris: PUF, 1972.

. Oeuvres. Paris: PUF, 1959.

CRARY, Alice. "Human, Animals, Right and Wrong". In: Wittgenstein and the Moral Life: Essays in Honor of Cora Diamond. Ed. Crary, Alice. Cambridge: MIT, p.381-404, 2007.

CURLE, Clinton. Humanité: John Humphrey's Alternative Account of Human Rights. Toronto: University of Toronto Press, 2007.

22 Je souhaite remercier Joanne Lefebvre et Benjamin Brice pour leur aide précieuse dans la préparation de cet essai. 
ENGLE, Sally Merry. Human Rights and Gender Violence: Translating International Law into Local Justice. Chicago: Chicago University Press, 2006.

GLENDON, Mary Ann. The Forum and the Tower: How Scholars and Politicians Have Imagined the World, from Plato to Eleanor Roosevelt. Oxford: Oxford University Press, 2011.

GUERLAC, Suzanne. Thinking in Time: An Introduction to Henri Bergson. Ithaca: Cornell University Press, 2006.

IGNATIEFF, Michael. Human Rights as Politics and Idolatry. Princeton Princeton University Press, 2003.

JAMES, William. Pragmatism. Cambridge: Harvard University Press, 2000.

JANKELEVITCH, Vladimir. Henri Bergson. Paris: PUF, 2008.

KATEB, George. Human Dignity. Cambridge: Harvard University Press, 2011.

LEFEBVRE, Alexandre. 'The Rights of Man and the Care of the Self. In: Political Theory: an International Journal of Political Philosophy, n.44 (4), 2016, p.518540.

. Human Rights as a Way of Life: On Bergson's Political Philosophy. Stanford: Stanford University Press, 2013.

. 'Mary Wollstonecraft, Human Rights, and the Care of the Self. In:

Humanity: an International Journal of Human Rights, Humanitarianism, and Development, n.7 (2), 2016, p.179-200.

MOYN, Samuel. The Last Utopia: Human Rights in History. Cambridge: Harvard University Press, 2010.

NUSSBAUM, Martha. Creating Capabilities: The Human Development Approach. Cambridge: Cambridge University Press, 2011.

; SUNSTEIN, Cass. Animal Rights: Current Debates and New Directions.

New York: Oxford University Press, 2005.

ORENTLICHER, Diane. "Religion and Relativism". In: Human Rights as Politics and Idolatry. Ed. Gutmann, Amy. Princeton: Princeton University Press, p.140-58, 2001.

PIPPEN, Robert B. "Hannah Arendt and the Bourgeois Origins of Totalitarian Evil". In: The Persistence of Subjectivity. Cambridge: Cambridge University Press, p.146-167, 2005.

REGAN, Tom. The Case for Animal Rights. Berkeley: University of California Press, 2004. 
ROSEN, Michael. Dignity: its History and Meaning. Cambridge: Harvard University Press, 2012.

SCHMITT, Carl. Théologie politique. Paris: Gallimard, 1988.

SINGER, Peter. Animal Liberation. 4th ed. London: Pimlico, 1995.

SOULEZ, Philippe. Bergson politique. Paris: PUF, 1989. ; WORMS, Frédéric. Bergson. Paris: PUF, 2002.

WIT'TE, John; GREEN, M. Christine (eds). Religion and Human Rights: An Introduction. Oxford: Oxford University Press, 2011.

WORMS, Frédéric. Bergson ou les deux sens de la vie. Paris: PUF, 2004. 